\title{
Upregulation of microRNA-492 induced by epigenetic drug treatment inhibits the malignant phenotype of clear cell renal cell carcinoma in vitro
}

\author{
AIBING WU*, KUNPENG WU*, MINGCHUN LI*, LINGLI BAO, XIANG SHEN, \\ SHUNJUN LI, JINMEI LI and ZHIXIONG YANG
}

Oncology Center, The Affiliated Hospital of Guangdong Medical College, Zhanjiang, Guangdong 524001, P.R. China

Received May 15, 2014; Accepted February 13, 2015

DOI: $10.3892 / \mathrm{mmr} .2015 .3550$

\begin{abstract}
Clear cell renal cell carcinoma (ccRCC) is the most common type of cancer of the renal parenchyma. MicroRNAs (miRNAs) are non-coding RNAs of $\sim 22$ nucleotides in length, which function as post-transcriptional regulators. Recently, the downregulation of miRNA (miR)-492 was observed to be associated with ccRCC; however, the molecular mechanism by which miR-492 inhibited ccRCC remained to be elucidated. In the present study, it was demonstrated that miR-492 was markedly downregulated in ccRCC tissues when compared with adjacent normal tissues, as determined by reverse transcription-quantitative poymerase chain reaction (PCR). This downregulation was predominantly due to the hypermethylation of the $\mathrm{CpG}$ island of the miR-492 promoter, which was detected by methylation specific PCR and bisulfite genomic sequencing PCR, and was shown to inhibit miR-492 transcription. Through the use of a DNA demethylation agent, 5-aza-2'-deoxycytidine or the histone deacetylase inhibitor 4-phenylbutyric acid, the expression level of miR-492 was significantly upregulated in ccRCC cells, which further inhibited cell proliferation and invasion, while promoting cell apoptosis and adhesion. In conclusion, the present study provided novel insights into the potential mechanisms involved in ccRCC and it is hypothesized that miR-492 may become a promising therapeutic agent in the treatment of ccRCC.
\end{abstract}

Correspondence to: Professor Zhixiong Yang, Oncology Center, The Affiliated Hospital of Guangdong Medical College, 57 Renmin Road, Zhanjiang, Guangdong 524001, P.R. China

E-mail: yangzhixiong068@163.com

${ }^{*}$ Contributed equally

Key words: clear cell renal cell carcinoma, proliferation, apoptosis, microRNA-492, adhesion, invasion

\section{Introduction}

Clear cell renal cell carcinoma (ccRCC) is the most common type of cancer of the renal parenchyma. Although current ccRCC treatment involves surgery combined with chemotherapy and radiotherapy, the median survival rate of patients with ccRCC remains low and a significant proportion of patients with ccRCC are at a high risk of relapse (1-3). Therefore, the development of effective therapeutic targets for ccRCC is urgently required.

MicroRNAs (miRNAs) are non-coding single-stranded RNAs between 19 and 25 nucleotides in length. They negatively regulate the expression of target genes by binding to target mRNAs at a post-transcriptional level. miRNAs are involved in various biological processes, including tumorigenesis, but may also function as tumor suppressors or promoters (4). Similarly to protein-coding genes, the expression of miRNAs may also be mediated by epigenetic processes, including DNA methylation, which may also be involved in the development and progression of human malignancies $(5,6)$.

miRNA (miR)-492, has been observed to be associated with multiple types of cancer, including retinoblastoma, hepatoblastoma, non-small cell lung cancer, pancreatic cancer and oropharyngeal carcinoma (7-14). Recently, it was reported that the expression levels of miR-492 were reduced in rectal cancer tissues compared with those of the normal rectal mucosa, suggesting that miR-492 may have an inhibitory role in the regulation of the development and progression of rectal cancer $(10,14)$. In addition, numerous downregulated miRNAs in cancer have been demonstrated to be associated with epigenetic mechanisms, for example hypermethylation of their promoters $(15,16)$. However, the detailed mechanism of miR-492 function, as well as the epigenetic regulatory processes in ccRCC have remained to be elucidated.

The present study aimed to investigate the epigenetic regulation of the expression of miR-492 in ccRCC. The effect of miR-492 on cell proliferation, apoptosis, invasion and adhesion in ccRCC cells was also examined.

\section{Materials and methods}

Reagents and materials. Dulbecco's modified Eagle's medium (DMEM), fetal bovine serum(FBS), TRIzol reagent, the TaqMan 
MicroRNA assay kit, the bicinchoninic acid (BCA) protein assay kit and Lipofectamine ${ }^{\circledR} 2000$ were purchased from Invitrogen Life Technologies (Carlsbad, CA, USA). The demethylation drug 5-Aza-2'-deoxycytidine (Aza) and the histone deacetylase inhibitor 4-phenylbutyric acid (PBA) were purchased from Sigma-Aldrich (St. Louis, MO, USA). The miRNeasy mini kit was purchased from Qiagen (Valencia, CA, USA). Mouse monoclonal anti-E-cadherin (1:500; cat. no. YM0208), mouse monoclonal Vimentin(1:500; cat. no. YM0645), rabbit polyclonal Caspase 3 (1:500; cat. no. YT0656), mouse monoclonal BH3 interacting-domain death agonist (Bid; 1:500; cat. no. YM0062), rabbit polyclonal phosphorylated B-cell lymphoma-2 (Bcl-2; 1:500; cat. no. YP0031) and mouse monoclonal $\beta$-actin (1:500; cat. no. YM3028) antibodies were purchased from ImmunoWay (Cambridge, UK). Goat anti-mouse (1:1,000; cat. no. SA001) and goat anti-rabbit (1:1,000; cat. no. SA009) $\operatorname{IgG}(\mathrm{H}+\mathrm{L})-\mathrm{HRP}$ secondary antibodies were purchased from Auragene Bioscience (Changsha, China). A cell invasion assay kit was purchased from Merck Millipore (Darmstadt, Germany).

Tissue collection. All protocols in the present study were approved by the Ethics Committee of Central South University (Changsha, China). Written informed consent was obtained from all patients with ccRCC included in the study. A total of six ccRCC tissues and matched adjacent normal tissues were collected at the Department of Nephrology, Xiangya Hospital of Central South University. None of the patients had received a blood transfusion, radiotherapy or chemotherapy prior to the surgery. All samples were immediately snap-frozen in liquid nitrogen (Auragene Bioscience) following surgical removal and stored at $-80^{\circ} \mathrm{C}$ until further use.

Cell culture. The present study utilized five human ccRCC cell lines, 786-O, ACHN, SN12C, A704 and TK10, as well as a normal renal cell line, HEK293, which were obtained from the Cell Bank of Central South University. Cells were cultured in DMEM with $10 \%$ FBS in a humidified atmosphere containing $5 \% \mathrm{CO}_{2}$ at $37^{\circ} \mathrm{C}$.

RNA extraction and miRNA expression assay. miRNAs were isolated from tissues or cells using the miRNeasy mini kit according to the manufacturer's instructions. The miRNA expression was then determined via reverse transcription quantitative polymerase chain reaction (RT-qPCR) using the TaqMan MicroRNA assay kit on a 7500 Fast Real Time PCR system (Applied Biosystems Life Technologies, Foster City, CA, USA), in accordance with the manufacturer's instructions. U6 was used as an endogenous control. For each sample, three independent experiments were performed. The relative expression levels of mRNA and miRNA were analyzed using the $2^{-\Delta \Delta C t}$ method (17).

Measurement of miR-492 promoter CpG island methylation status using bisulfite genomic sequencing PCR (BSP). Genomic DNA was extracted from ccRCC 786-O and ACHN cells using a genomic DNA extraction kit (Takara, Dalian, China). Genomic DNA (1 $\mu \mathrm{g})$ was modified with bisulfite using the Epitect bisulfite kit (Qiagen) according to the manufacturer's instructions, and eluted in a total of $40 \mathrm{ml}$ elution buffer (Auragene Bioscience). Subsequently,
$2 \mathrm{ml}$ modified DNA was used as the template for the BSP reaction. The primer sequences were as follows: miR-429 forward, 5'-GTGACCTGGCTCCAGGAAAGGC-3', and reverse, 5'-CAGATGGAAAAGATGAAACAATGGG-3'. The PCR cycling conditions were set at: $94^{\circ} \mathrm{C}$ for $4 \mathrm{~min}$, followed by 35 cycles at $94^{\circ} \mathrm{C}$ for $30 \mathrm{sec}, 55^{\circ} \mathrm{C}$ for $30 \mathrm{sec}, 72^{\circ} \mathrm{C}$ for $5 \mathrm{~min}$, and a final step at $72^{\circ} \mathrm{C}$ for $5 \mathrm{~min}$. The PCR products were gel purified, cloned into the pUC18-T plasmid (Sangon Biotech, Shanghai, China) and subsequently sequenced by BGI (Wuhan, China).

Measurement of miR-492 promoter $C p G$ island methylation status using methylation specific PCR (MS-PCR). Genomic DNA was extracted using a genomic DNA extraction kit (Takara). Genomic DNA (1 $\mu \mathrm{g})$ was modified with bisulfite using the Epitect Bisulfite kit (Qiagen), according to the manufacturer's instructions and eluted in a total of $40 \mathrm{ml}$ elution buffer. MS-PCR was performed on bisulfate-treated DNA. The primer sequences were as follows: Methylated miR-492 forward, 5'-CGGGGATATTATCGAGGTATATTC-3' and reverse, 5'-AACTAACACAAACCCTCTACCG-3' and unmethylated miR-492 forward, 5'-TGGGGATATTATTGAG GTATATTTG-3' and reverse, 5'-AAACTAACACAAACC CTCTACCAC-3'. The PCR cycling conditions were set at: $94^{\circ} \mathrm{C}$ for $4 \mathrm{~min}$, followed by 35 cycles at $94^{\circ} \mathrm{C}$ for $30 \mathrm{sec}, 55^{\circ} \mathrm{C}$ for $30 \mathrm{sec}, 72^{\circ} \mathrm{C}$ for $5 \mathrm{~min}$, and a final step at $72^{\circ} \mathrm{C}$ for $5 \mathrm{~min}$.

Western blot analysis. Western blotting was used to examine the protein expression levels in each group. Cells were lysed in cold radioimmunoprecipitation buffer (Auragene Bioscience). The BCA Protein assay kit was used to determine the protein concentration and was used in accordance with the manufacturer's instructions. Subsequently, the proteins were separated by $10 \%$ SDS-PAGE (Auragene Bioscience) and transferred onto a polyvinylidene difluoride (PVDF) membrane (Auragene Bioscience). The PVDF membrane was blocked in 5\% nonfat dried milk in phosphate-buffered saline (PBS) for $4 \mathrm{~h}$. Subsequently, the PVDF membrane was incubated with specific primary antibodies at $37^{\circ} \mathrm{C}$ for $3 \mathrm{~h}$. Following three washes in PBS, each for $5 \mathrm{~min}$, the PVDF membrane was incubated with the appropriate secondary antibody at $37^{\circ} \mathrm{C}$ for $1 \mathrm{~h}$. Following a further three washes in PBS, each for $5 \mathrm{~min}$, an enhanced chemiluminescence western blotting kit (Thermo Fisher Scientific, Rockford, IL, USA) was used to detect the immune complexes on the PVDF membrane.

Epigenetic drug treatment of cells. The human ccRCC cell lines, 786-O and ACHN were treated with Aza (15.55 nM), or PBA $(1.5 \mathrm{nM})$ or both in combination, with or without anti-miR-429 (20 $\mu \mathrm{M})$ (HmiR-AN0497; Genecopoeia, Guangzhou, China), for $72 \mathrm{~h}$.

Cell counting kit (CCK)-8 cell proliferation assay. CCK-8 was used to evaluate cell proliferation. A total of $5 \times 10^{3}$ cells were seeded in 96-well plates for $24 \mathrm{~h}$, treated with the indicated drugs and further incubated for 0, 24, 48 and $72 \mathrm{~h}$, respectively. At $1 \mathrm{~h}$ prior to the completion of the incubation, $10 \mu \mathrm{lCCK}-8$ was added to each well. The optical density at $450 \mathrm{~nm}$ in each well was determined using an enzyme immunoassay analyzer (Multiskan M3; Thermo Fisher Scientific). 
A

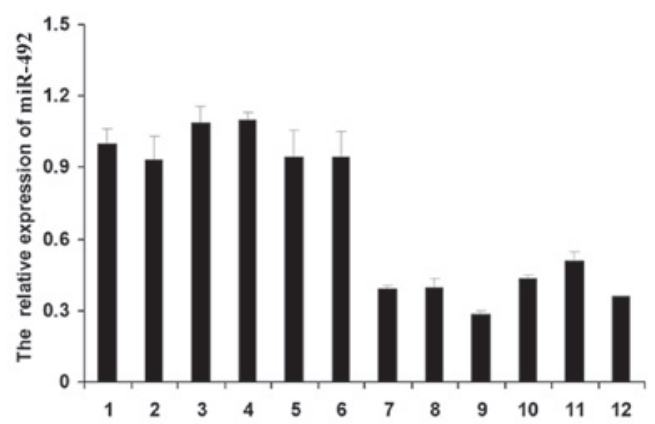

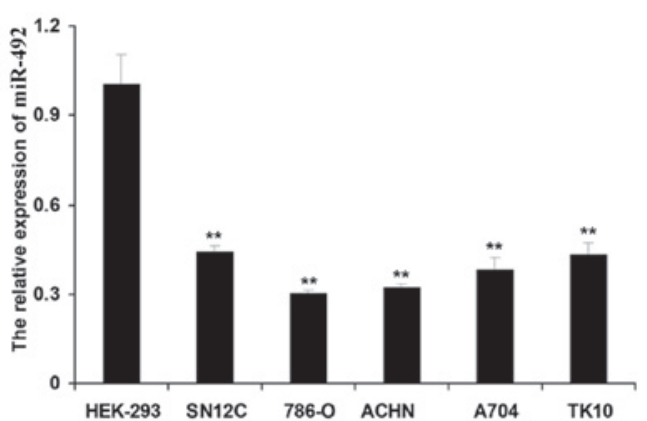

HEK-293 SN12C 786-O ACHN A704 TK10

B

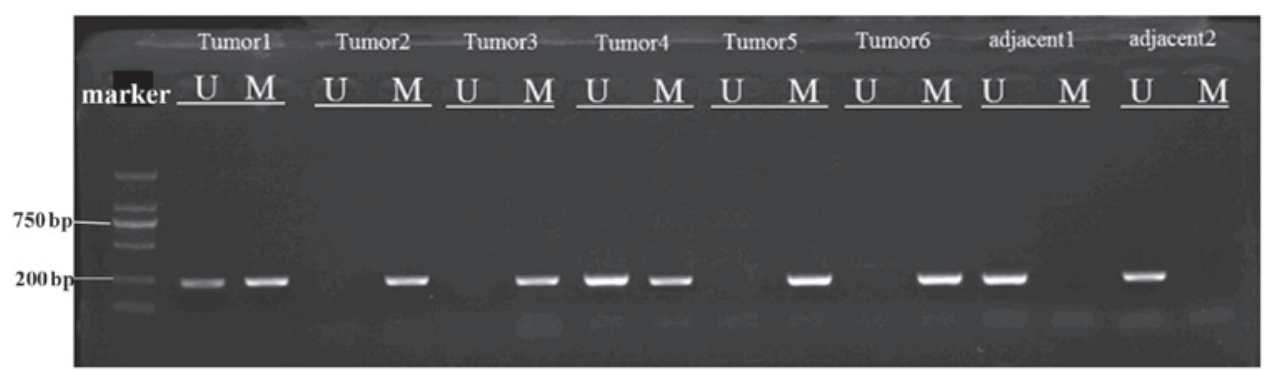

Figure 1. miR-492 expression is downregulated in ccRCC cells and tissues. (A) Reverse transcription quantitative PCR was performed to determine the relative expression of miR-492 in six matched adjacent normal tissues (1-6) and ccRCC tissues (7-12). The relative expression of miR-492 was also examined in five ccRCC cell lines, 786-O, ACHN, SN12C, A704 and TK10, as well as a normal renal cell line HEK293. ${ }^{* *} \mathrm{P}<0.01$, vs. HEK293. (B) Methylation specific PCR was used to determine the methylation status in the CpG island of the miR-492 promoter in six ccRCC tissues (Tumor 1-6) as well as in two adjacent normal tissues (adjacent 1-2). U, unmethylated; M, methylated; PCR, polymerase chain reaction; ccRCC, clear cell renal cell carcinoma; miR, microRNA; bp, basepairs.

MTT cell proliferation assay. For all groups, $10 \times 10^{3}$ cells/well were plated in a 96-well plate and incubated for 0, 24, 48 and $72 \mathrm{~h}$, respectively, at $37^{\circ} \mathrm{C}$ with $5 \% \mathrm{CO}_{2}$. To assess cell proliferation, $50 \mu \mathrm{l}$ MTT ( $5 \mathrm{mg} / \mathrm{ml}$; Auragene Bioscience) in PBS was added and cells were then incubated for $4 \mathrm{~h}$ at $37^{\circ} \mathrm{C}$ and $5 \% \mathrm{CO}_{2}$. Subsequently, the supernatant was removed and $150 \mu 1$ dimethyl sulfoxide (Auragene Bioscience) was added. The absorbance was detected at $450 \mathrm{~nm}$ with a Microplate Reader (Model 680 XR; Bio-Rad Laboratories, Inc., Hercules, CA, USA).

Cell apoptosis assay. Flow cytometry was used to determine the level of cell apoptosis. At $24 \mathrm{~h}$ post-transfection, cells were harvested and washed twice with cold PBS.

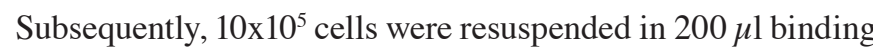
buffer with $10 \mu \mathrm{l}$ Annexin-V-fluorescein isothiocyanate and $5 \mu \mathrm{l}$ propidium iodide-phycoerythrin and incubated in the dark for $30 \mathrm{~min}$. Following this stage, $300 \mu \mathrm{l}$ binding buffer (Keygentec Biotech. Co., Ltd., Nanjing, China) was added and cells were subjected to flow cytometric analysis (Moflo XDP; Beckman Coulter, Krefeld, Germany).

Cell invasion assay. Cells were administered the indicated drug treatments for $72 \mathrm{~h}$, starved in serum-free medium for $24 \mathrm{~h}$ and then resuspended in serum-free medium. The cells were added to the upper chamber of a transwell (Transwell kit; BD Biosciences, Bedford, MA, USA), while the lower chamber was filled with base medium containing $10 \%$ FBS. Following incubation for $24 \mathrm{~h}$, cells attached to the bottom of the chamber were stained with crystal violet (Auragene Bioscience) for $20 \mathrm{~min}$ and then washed and air-dried. Invasive cells were observed under a microscope (AE31; Motic, Fujian, China).
Cell adhesion assay. Cells administered with the indicated drug treatments for $72 \mathrm{~h}$ were seeded into a 24 -well plate and $500 \mu \mathrm{l}$ fibronectin $(20 \mu \mathrm{g} / \mathrm{ml}$; Sigma-Aldrich) was added into each well. The 24 -well plate was incubated at $4^{\circ} \mathrm{C}$ overnight. Following washing with PBS, $1 \%$ bovine serum albumin (Auragene Bioscience) was added to each well for blocking at $37^{\circ} \mathrm{C}$ for $2 \mathrm{~h}$. Subsequently, the blocking solution was removed and the cells were dried. Cells were then digested and centrifuged at $200 \mathrm{x} \mathrm{g}$ for $5 \mathrm{~min}$. Serum-free medium (HyClone, Beijing, China) was used to produce a single-cell suspension and the cell concentration was adjusted to $10^{6}$ cells $/ \mathrm{ml}$. The cell suspension $(1 \mathrm{ml})$ was added into each well with a slide (Auragene Bioscience) and then incubated at $37^{\circ} \mathrm{C}$ with $5 \% \mathrm{CO}_{2}$ for $2 \mathrm{~h}$. Following the removal of non-adherent cells using PBS, $4 \%$ neutral formalin (Auragene Bioscience) was used to fix adherent cells for $3 \mathrm{~h}$ and each well was treated with $500 \mu \mathrm{l}$ crystal violet and incubated for $2 \mathrm{~h}$. Adherent cells were then observed under a microscope (AE31; Motic).

Statistical analysis. Data are expressed as the mean \pm standard deviation of three independent experiments and were analyzed using SPSS 17.0 statistical software (SPSS, Inc., Chicago, IL, USA). The differences between groups were determined using a one-way analysis of variance. $\mathrm{P}<0.05$ was considered to indicate a statistically significant difference.

\section{Results}

Expression of miR-492 is markedly downregulated in ccRCC tissues and cells. The expression levels of miR-492 in ccRCC tissues and cells, as well as in their matched adjacent tissues and normal renal cells were examined. As shown in Fig. 1A, the expression of miR-492 was significantly reduced in ccRCC 

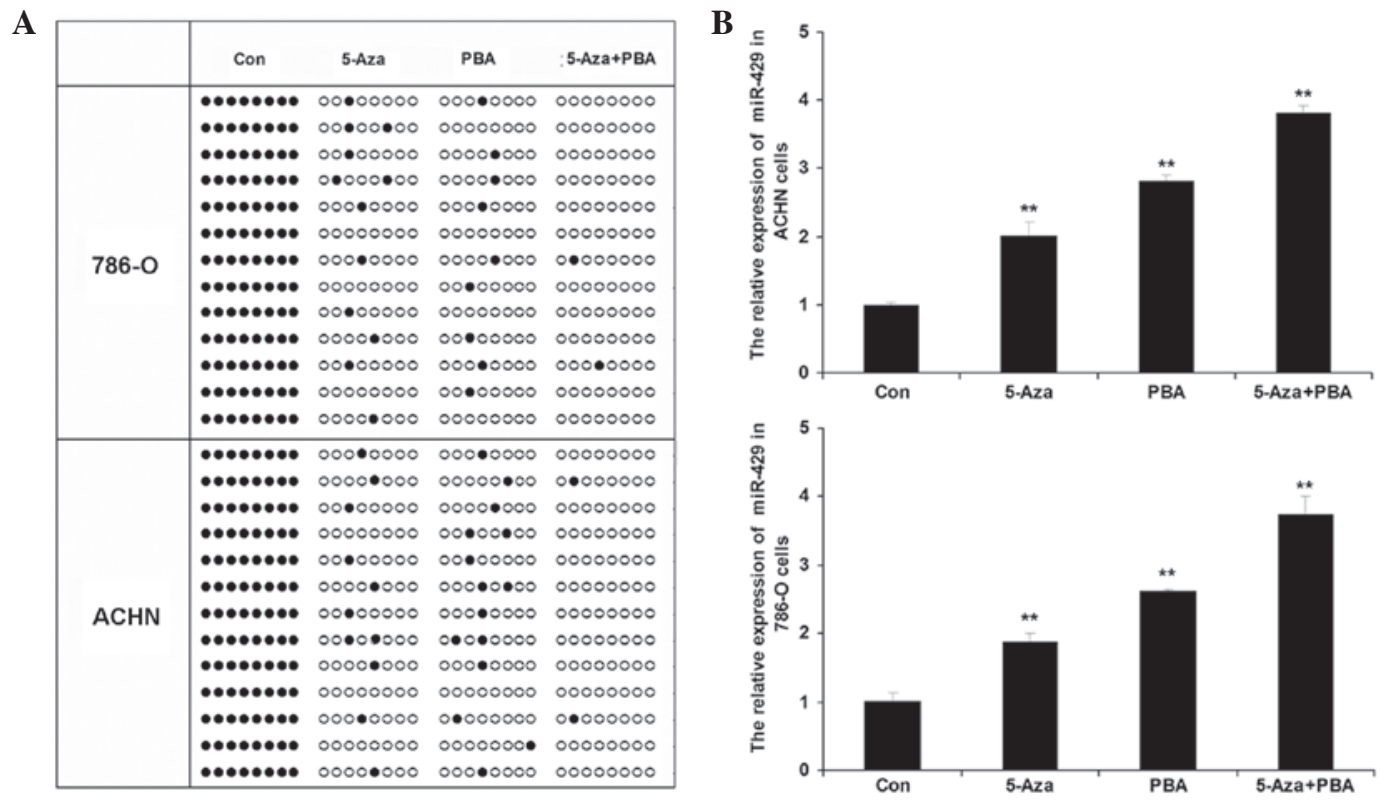

Figure 2. Epigenetic drug treatment induces upregulation of miR-492 expression in ccRCC cells. (A) Bisulfite genomic sequencing PCR was used to determine the methylation status in the $\mathrm{CpG}$ island of the miR-492 promoter in two ccRCC cell lines, 786-O and ACHN, treated with Aza (15.55 nM), PBA (1.5 nM) or Aza $(15.55 \mathrm{nM})+$ PBA $(1.5 \mathrm{nM})$ for $72 \mathrm{~h}$. Black dots indicate unmethylated sites and white circles indicate methylated sites. (B) Reverse transcription quantitative PCR was performed to determine the relative expression of miR-492 in ccRCC 786-O and ACHN cells treated with Aza (15.55 nM), PBA (1.5 nM) or Aza $(15.55 \mathrm{nM})+\mathrm{PBA}(1.5 \mathrm{nM})$ for $72 \mathrm{~h}$. Con, cells without any treatment. ${ }^{* *} \mathrm{P}<0.01$, vs. Con. PCR, polymerase chain reaction; ccRCC, clear cell renal cell carcinoma; miR, microRNA; Con, control; Aza, 5-Aza-2'-deoxycytidine; PBA, 4-phenylbutyric acid.

A
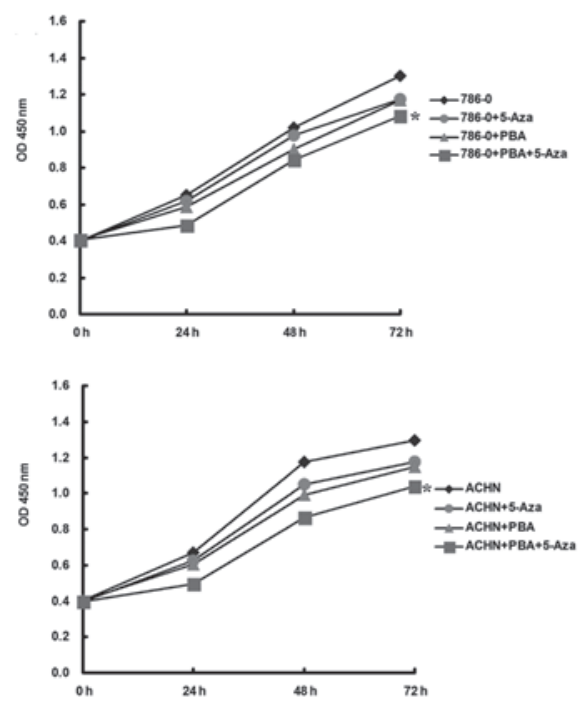

B
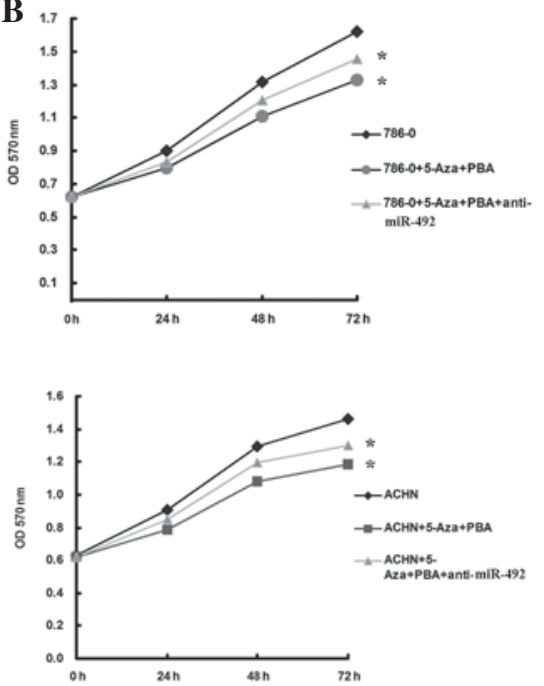

Figure 3. Upregulation of miR492 induced by epigenetic drug treatment inhibits ccRCC cell proliferation. (A) CCK-8 cell proliferation assay was performed to determine the cell proliferation in ccRCC 786-O and ACHN cells treated with Aza (15.55 nM), PBA (1.5 nM) or Aza (15.55 nM) + PBA (1.5 nM) for $72 \mathrm{~h}$. (B) MTT cell proliferation assay was performed to determine the cell proliferation in ccRCC cell lines 786-O and ACHN treated with Aza (15.55 nM) + PBA $(1.5 \mathrm{nM})$ for $72 \mathrm{~h}$, with or without transfection with anti-miR-492. "P $<0.05$, vs. relative control $786-\mathrm{O}$ or ACHN group. ccRCC, clear cell renal cell carcinoma; miR, microRNA; Con, control; Aza, 5-Aza-2'-deoxycytidine; PBA, 4-phenylbutyric acid; CCK-8, cell counting kit-8.

tissues compared with that of their matched adjacent tissues. In addition, analogous results were observed in the ccRCC cell lines, 786-O, ACHN, SN12C, A704 and TK10, compared with HEK 293 cells. The methylation status in the $\mathrm{CpG}$ island of the miR-492 promoter, which may be involved in the downregulation of miR-492, was further investigated. MS-PCR was used to determine the methylation status in the $\mathrm{CpG}$ island of the miR-492 promoter in six ccRCC tissues, as well as two normal adjacent tissues. As shown in Fig. 1B, tumor 1 and tumor 4 exhibited hemimethylation and tumors 2, 3, 5 and 6 exhibited methylation. Conversely, the two normal adjacent tissues were observed to be unmethylated. These findings suggested that hypermethylation of the miR-492 promoter may contribute to the downregulation of miR-492 in ccRCC.

Upregulation of miR-492 is induced by epigenetic drug treatment in ccRCC cells. Following treatment of the ccRCC cell lines, 786-O and ACHN, with Aza, PBA or Aza + PBA, the methylation status of the CpG island of the miR-492 promoter was determined using BSP. As shown in Fig. 2A, a 

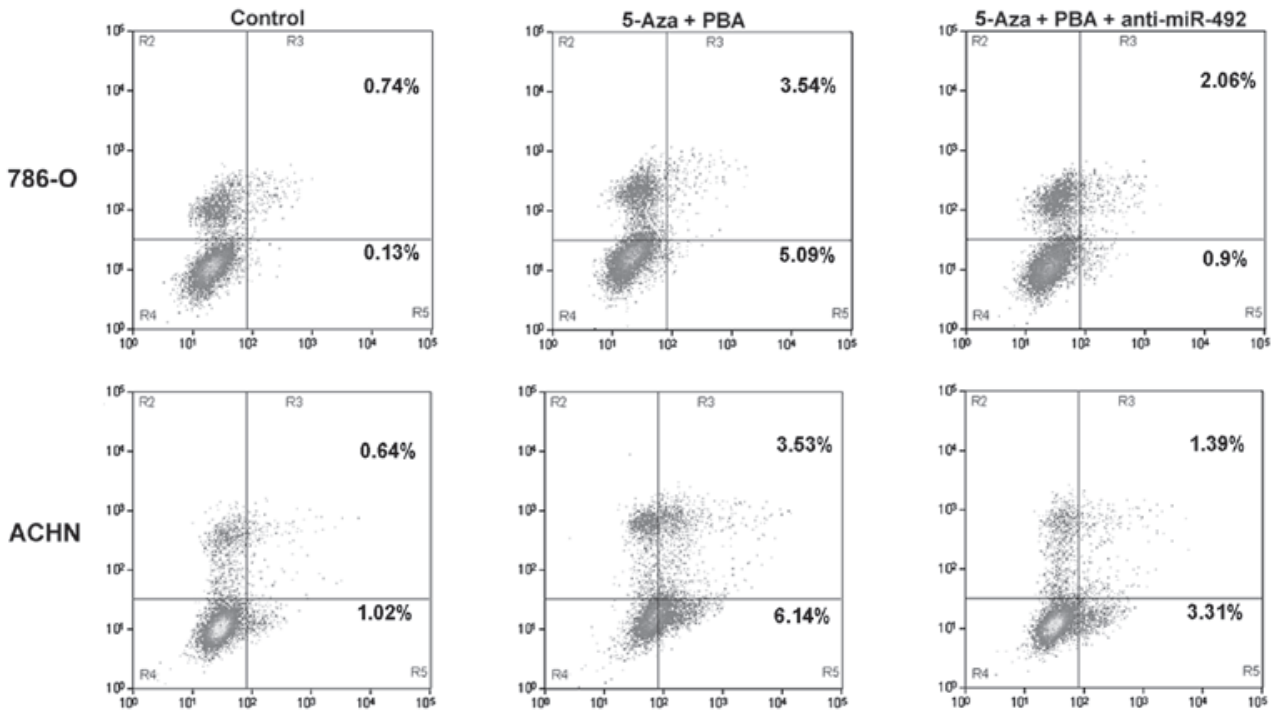

Figure 4. Cell apoptosis assay was performed to determine the cell apoptosis level in clear cell renal cell carcinoma 786-O and ACHN cells treated with Aza $(15.55 \mathrm{nM})+$ PBA $(1.5 \mathrm{nM})$ for $72 \mathrm{~h}$, with or without transfection with anti-miR-492. Control, cells without any treatment. Aza, 5-Aza-2'-deoxycytidine; PBA, 4-phenylbutyric acid; miR, microRNA.

A

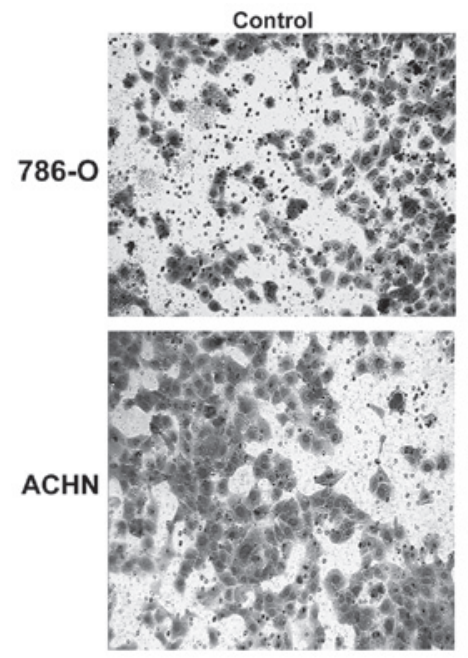

B

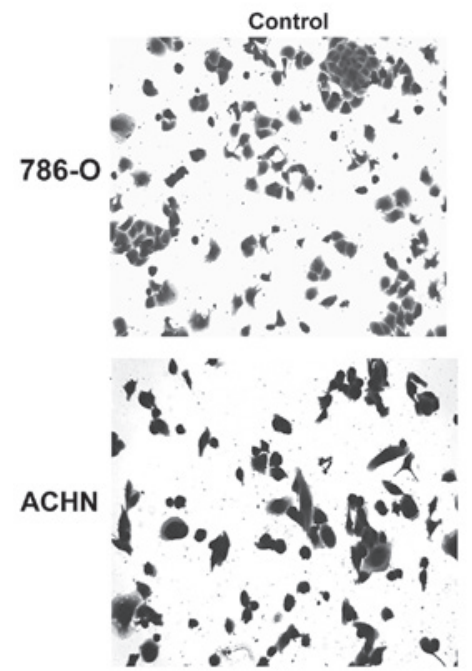

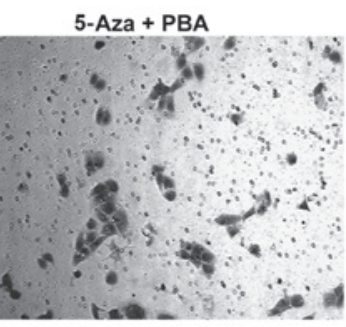

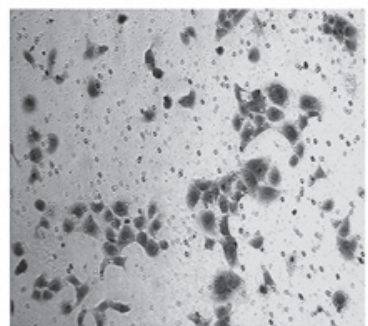

5-Aza + PBA
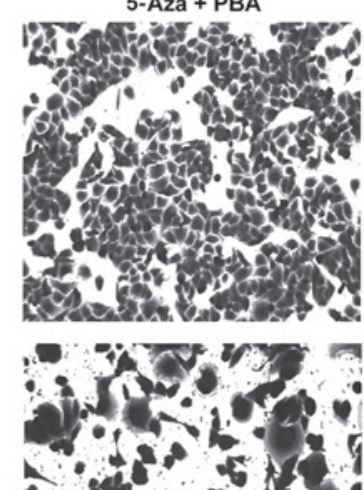

\section{.}

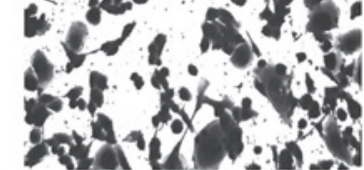

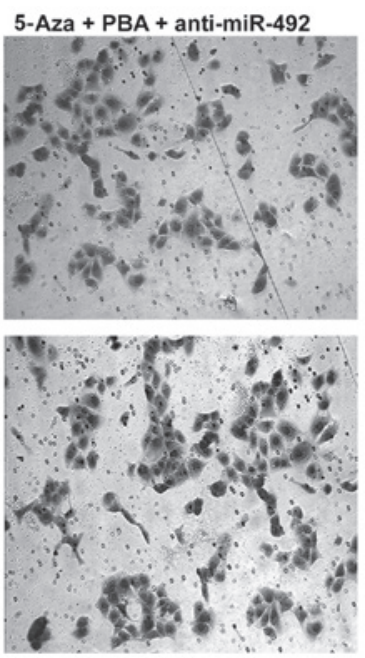

5-Aza + PBA + anti-miR-492

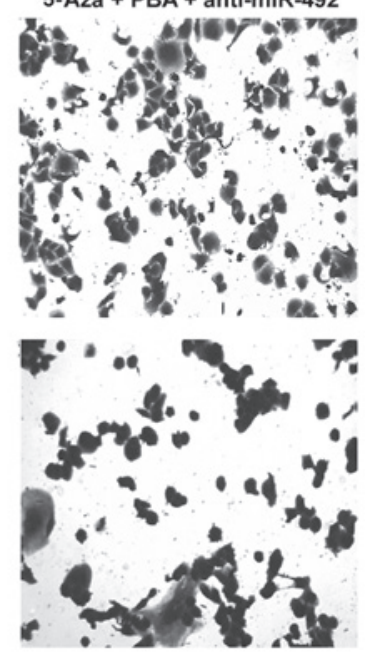

Figure 5. Upregulation of miR-492 inhibits invasion and enhances adhesion in ccRCC cells. (A) A cell invasion assay was performed to determine the cell invasion level in ccRCC 786-O and ACHN cells treated with Aza (15.55 nM) + PBA (1.5 nM) for $72 \mathrm{~h}$, with or without transfection with anti-miR-492. Cells were stained with crystal violet (magnification, x200). (B) A cell adhesion assay was performed to determine the cell adhesion level in ccRCC 786-O and ACHN cells treated with Aza (15.55 nM) + PBA (1.5 nM) for $72 \mathrm{~h}$, with or without transfection with anti-miR-492. Cells were stained with crystal violet (magnification, x200). Control, cells without any treatment; ccRCC, clear cell renal cell carcinoma; miR, microRNA; Aza, 5-Aza-2'-deoxycytidine; PBA, 4-phenylbutyric acid. 


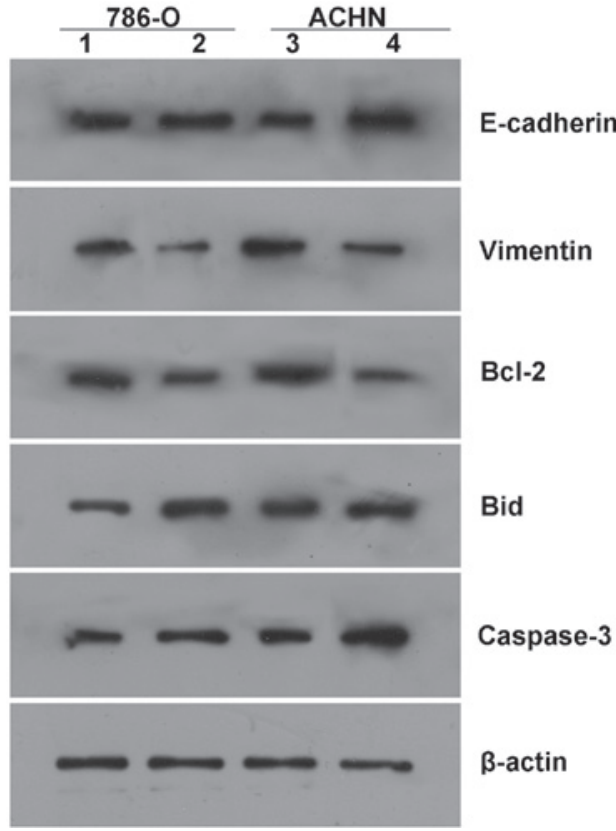

Figure 6. Western blotting was performed to determine the protein expression levels of several factors involved in cell apoptosis, adhesion and motility in clear cell renal cell carcinoma 786-O and ACHN cells. $\beta$-actin was used as an internal reference. 1 and 3 , cells without any treatment; 2 and 4 , cells treated with 5-Aza-2'-deoxycytidine $(15.55 \mathrm{nM})+4$-phenylbutyric acid $(1.5 \mathrm{nM})$ for $72 \mathrm{~h}$.

significantly higher level of methylation was observed in the 786-O and ACHN cells following treatment with Aza, PBA or Aza + PBA. In addition, the expression levels of miR-492 in each group were determined. As indicated in Fig. 2B, miR-492 expression was significantly upregulated following treatment with Aza and PBA, particularly following treatment with a combination of Aza + PBA.

Upregulation of miR-492 induced by epigenetic drug treatment inhibits proliferation in ccRCC cells. The effects of miR-492 upregulation on ccRCC cells were further investigated. As shown in Fig. 3A, the epigenetic drug-induced upregulation of miR-492 significantly inhibited the proliferation of ccRCC cells. To further confirm these findings, ccRCC cells were transfected with anti-miR-492, which is able to reverse the upregulation of miR-492 induced by treatment with Aza + PBA, and further MTT investigation revealed that cellular proliferation was enhanced in the Aza + PBA + anti-miR-492 group, when compared with that in the Aza + PBA group (Fig. 3B). These data suggested that miR-492 has a suppressive role in the regulation of ccRCC cell proliferation.

Upregulation of miR-492 induced by epigenetic drug treatment promotes apoptosis in ccRCC cells. The effect of miR-492 on apoptosis was determined in the ccRCC cell lines, 786-O and ACHN. As shown in Fig. 4, the upregulation of miR-492 induced by treatment with Aza + PBA significantly promoted apoptosis in the ccRCC cell lines, 786-O and ACHN. However, transfection with anti-miR-492 markedly attenuated this effect. These findings suggested that miR-492 upregulation was able to induce ccRCC cell apoptosis.
Upregulation of miR-492 induced by epigenetic drug treatment inhibits invasion while promoting adhesion in ccRCC cells. As shown in Fig. 5A, the upregulation of miR-492 induced by epigenetic drug treatment significantly inhibited the invasion of the ccRCC cell lines, 786-O and ACHN, which was attenuated by transfection with anti-miR-492. Furthermore, miR-492 upregulation induced by treatment with Aza + PBA markedly promoted cell adhesion in the ccRCC cell lines, 786-O and ACHN, which was also inhibited by transfection with anti-miR-492 (Fig. 5B). These findings suggested that miR-492 upregulation was able to inhibit invasion while promoting adhesion in ccRCC cells.

Changes in gene expression following upregulation of miR-492 in ccRCC cells. To further investigate the molecular mechanism underlying the effects of miR-492 in ccRCC cells, the expression of several key factors associated with cell survival and adhesion in ccRCC cells were examined following treatment with epigenetic drugs. As shown in Fig. 6, the upregulation of miR-492 expression induced by treatment with Aza + PBA promoted the expression of pro-adhesive E-cadherin, and inhibited the expression of anti-adhesive Vimentin, consistent with the aforementioned data demonstrating that miR-492 upregulation promoted adhesion in ccRCC cells. In addition, upregulation of miR-492 also inhibited the expression of the anti-apoptotic protein $\mathrm{Bcl}-2$, and enhanced the expression of pro-apoptotic Bid and Caspase 3, which was consistent with the findings that miR-492 upregulation promoted ccRCC cell apoptosis.

\section{Discussion}

In the present study, it was demonstrated that the expression levels of miR-492 were significantly downregulated in ccRCC tissues and cells, when compared with those of normal renal tissues and cells. In addition, this downregulation was accompanied by hypermethylation of the $\mathrm{CpG}$ island of the miR-492 promoter. Furthermore, treatment with epigenetic drugs markedly promoted the expression of miR-492 in ccRCC cells. Further investigation demonstrated that the upregulation of miR-492 induced by epigenetic drug treatment markedly inhibited proliferation and invasion, whilst promoting apoptosis and adhesion in ccRCC cells, suggesting that miR-492 has a suppressive role in the regulation of malignant phenotypes in ccRCC cells.

The detailed role of miR-492 in cancer has remained elusive. The deregulation of miR-492 has been previously reported in retinoblastoma; Zhao et al (7) revealed that miR-492 was highly expressed in retinoblastoma, suggesting that miR-492 may be involved in the tumorigenesis of retinoblastoma. Subsequently, von Frowein et al (8) reported that miR-492 was markedly upregulated in metastatic hepatoblastoma, suggesting that miR-492 may promote certain processes involved in the regulation of hepatoblastoma metastasis. Additionally, miR-492 was also reported to be associated with non-small cell lung cancer, pancreatic cancer and oropharyngeal carcinoma (11-13). In the present study, it was revealed that miR-492 was significantly downregulated in ccRCC tissues and five ccRCC cell lines. Gaedcke et al (10) also observed a decrease in miR-492 expression in rectal cancer tissues. 
In addition, $\mathrm{Wu}$ et al (14) revealed that miR-492 expression was downregulated in ccRCC tissues. However, details of the specific mechanisms underlying the involvement of miR-492 in ccRCC remains to be elucidated.

Similarly to protein-coding genes, epigenetic mechanisms, including DNA methylation and histone acetylation have also been revealed to be involved in the regulation of miRNA transcription (16). It has been well-established that DNA methylation in the $\mathrm{CpG}$ island of the gene promoter is the most common epigenetic modification observed in eukaryotic genomes (18) and hypermethylation may lead to decreased gene transcription (19). However, to the best of our knowledge, the epigenetic mechanisms by which the expression of miR-492 is mediated have not previously been investigated with regards to cancer. In the present study, the methylation level in the $\mathrm{CpG}$ island of the miR-492 promoter was significantly upregulated in ccRCC tissues. However, the normal renal tissues were observed to be unmethylated. As hypermethylation in the gene promoter has an inhibitory role in the regulation of gene transcription, it was hypothesized that hypermethylation of the miR-492 promoter may contribute to the downregulation of miR-492, as well as the development and progression of ccRCC. To further confirm the involvement of an epigenetic mechanism in the downregulation of miR-492, the ccRCC cell lines, 786-O and ACHN, were treated with two common epigenetic drugs, Aza and PBA. Aza is a DNA methyltransferase inhibitor, which is able to induce DNA demethylation, while PBA is a histone deacetylase inhibitor, which may induce histone acetylation (20,21). DNA demethylation and histone acetylation promote gene transcription $(22,23)$. The present data revealed that treatment with these epigenetic drugs significantly promoted the expression of miR-492, particularly when administered in combination, indicating that the expression levels of miR-492 in ccRCC were tightly regulated by epigenetic modulations, including DNA methylation and histone acetylation.

In addition, the upregulation of miR-492 induced in ccRCC cells treated with Aza and PBA resulted in a significant decrease in the proliferation of ccRCC cells. Accordingly, it was hypothesized that miR-492 may have an inhibitory role in the regulation of ccRCC cell proliferation. To verify this hypothesis, anti-miR-492 was applied, and the results demonstrated that anti-miR-492 reversed the inhibitory effects of Aza and PBA on ccRCC cell proliferation. Subsequently, the effects of miR-492 upregulation on ccRCC cell apoptosis, invasion and adhesion were further examined. The present data demonstrated that miR-492 upregulation significantly promoted cell apoptosis and adhesion, while suppressing cell invasion in ccRCC cells.

Furthermore, upregulation of apoptosis was accompanied by an increase in the expression of Caspase 3 and Bid, as well as a decrease in the expression of Bcl-2. Bcl-2 and Bid are two key members of the Bcl-2 family, which is involved in the regulation of cell survival. However, Bcl-2 and Bid have opposing effects on cell survival rate, since Bcl-2 is a key anti-apoptotic factor, whereas Bid functions as a death agonist able to promote cell apoptosis through heterodimerization with Bcl-2 $(24,25)$. In addition, sequential activation of the caspases is crucial in the execution phase of cell apoptosis, and Caspase 3 is a key executor (26,27). Accordingly, the present data suggested that these three key apoptosis-associated proteins may act as downstream effectors of miR-492 in ccRCC cells. In addition, the upregulation of adhesion and inhibition of invasion observed were consistent with the increased expression of E-cadherin as well as the reduced expression of Vimentin. E-cadherin is a cell-cell adhesion molecule and its increased expression may lead to upregulation of adhesion as well as inhibition of cell motility $(28,29)$. By contrast, Vimentin functions as a cytoskeletal linker protein and is critical in the regulation of cell motility $(30,31)$. In accordance with these results, it was suggested that miR-492 upregulation promoted adhesion while suppressing invasion in ccRCC cells, partially at least, through promoting the expression of E-cadherin and inhibiting the expression of Vimentin.

In conclusion, the present study revealed that miR-492 was markedly downregulated in ccRCC cells due to the hypermethylated status of the miR-492 promoter. In addition, the upregulation of miR-492 induced by epigenetic drug treatment inhibited cell proliferation and invasion, while it promoted cell apoptosis and adhesion in ccRCC cells. Based on these results, it was hypothesized that miR-492 has an inhibitory role in ccRCC, and may be a novel diagnostic or therapeutic target for ccRCC.

\section{Acknowledgements}

The present study was supported by funding from National Natural Science Foundation of China (grant no. 81201672), Research Fund for the Doctoral Program of Guangdong Medical College (grant no. XB1331) and Research Fund for the Doctoral Program of Affiliated Hospital of Guangdong Medical College (grant no. BK201208).

\section{References}

1. Diamond E, Riches J, Faltas B, Tagawa ST and Nanus DM: Immunologics and chemotherapeutics for renal cell carcinoma. Semin Intervent Radiol 31: 91-97, 2014.

2. Rydzanicz M, Wrzesiński T, Bluyssen HA and Wesoly J: Genomics and epigenomics of clear cell renal cell carcinoma: recent developments and potential applications. Cancer Lett 341: 111-126, 2013.

3. Chowdhury S, Matrana MR, Tsang C, Atkinson B, Choueiri TK and Tannir NM: systemic therapy for metastatic non-clear-cell renal cell carcinoma: recent progress and future directions. Hematol Oncol Clin North Am 25: 853-869, 2011.

4. Garg D and Cohen SM: miRNAs and aging: A genetic perspective. Ageing Res Rev 2014: 3-8, 2014.

5. Choi JD and Lee JS: Interplay between epigenetics and genetics in cancer. Genomics Inf 11: 164-173, 2013.

6. Watanabe K and Takai D: Disruption of the expression and function of microRNAs in lung cancer as a result of epigenetic changes. Front Genet 4: 275, 2013.

7. Zhao JJ, Yang J, Lin J, et al: Identification of miRNAs associated with tumorigenesis of retinoblastoma by miRNA microarray analysis. Childs Nerv Syst 25: 13-20, 2009.

8. von Frowein J, Pagel P, Kappler R, von Schweinitz D, Roscher A and Schmid I: MicroRNA-492 is processed from the keratin 19 gene and up-regulated in metastatic hepatoblastoma. Hepatology 53: 833-842, 2011.

9. Yoon KA, Yoon H, Park S, et al: The prognostic impact of microRNA sequence polymorphisms on the recurrence of patients with completely resected non-small cell lung cancer. J Thorac Cardiovasc Surg 144: 794-807, 2012.

10. Gaedcke J, Grade M, Camps J, et al: The rectal cancer microRNAome - microRNA expression in rectal cancer and matched normal mucosa. Clin Cancer Res 18: 4919-4930, 2012. 
11. Schultz NA, Werner J, Willenbrock H, et al: MicroRNA expression profiles associated with pancreatic adenocarcinoma and ampullary adenocarcinoma. Mod Pathol 25: 1609-1622, 2012.

12. Hui $\mathrm{AB}, \mathrm{Lin} \mathrm{A}, \mathrm{Xu} \mathrm{W}$, et al: Potentially prognostic miRNAs in HPV-associated oropharyngeal carcinoma. Clin Cancer Res 19: 2154-2162, 2013.

13. Kupcinskas J, Wex T, Link A, et al: Gene polymorphisms of micrornas in Helicobacter pylori-induced high risk atrophic gastritis and gastric cancer. PLoS One 9: e87467, 2014.

14. Wu X, Weng L, Li X, et al: Identification of a 4-microRNA signature for clear cell renal cell carcinoma metastasis and prognosis. PLoS One 7: e35661, 2012.

15. Kim JG, Kim TO, Bae JH, et al: Epigenetically regulated MIR941 and MIR1247 target gastric cancer cell growth and migration. Epigenetics 9: 1018-1030, 2014.

16. Suzuki H, Maruyama R, Yamamoto E and Kai M: Epigenetic alteration and microRNA dysregulation in cancer. Front Genet 4 258, 2013.

17. Hu Y, Liu J, Jiang B, et al: Mir-199a-5p loss up-regulated DDR 1 aggravated colorectal cancer by activating epithelial-to-mesenchymal transition related signaling. Dig Dis Sci 59: 2163-2172, 2014.

18. Shapiro JA: Epigenetic control of mobile DNA as an interface between experience and genome change. Front Genet 5: 87, 2014

19. Zhang Z, Chen Y, Tang J and Xie X: Frequent loss expression of and promotor hypermethylation in human cancers: a meta-analysis and systematic review. Pak J Med Sci 30: 432-437, 2014.

20. Izutsu N, Maesawa C, Shibazaki M, et al: Epigenetic modification is involved in aberrant expression of class III $\beta$-tubulin, TUBB3, in ovarian cancer cells. Int J Oncol 32: 1227-1235, 2008.

21. Song K, Han C, Zhang J, et al: Epigenetic regulation of MicroRNA-122 by peroxisome proliferator activated receptor-gamma and hepatitis b virus X protein in hepatocellular carcinoma cells. Hepatology 58: 1681-1692, 2013.
22. Fetahu IS, Höbaus J, Aggarwal A, et al: Calcium-sensing receptor silencing in colorectal cancer is associated with promoter hypermethylation and loss of acetylation on histone 3. Int J Cancer 135: 2014-2023, 2014

23. Xu Z, Li H and Jin P: Epigenetics-based therapeutics for neurodegenerative disorders. Curr Transl Geriatr Exp Gerontol Rep 1: 229-236, 2012.

24. Wang $\mathrm{Y}$ and Tjandra N: Structural insights of tBid, the caspase-8-activated Bid, and its BH3 domain. J Biol Chem 288: 35840-35851, 2013.

25. Renault TT and Chipuk JE: Getting away with murder: how does the BCL-2 family of proteins kill with immunity? Ann NY Acad Sci 1285: 59-79, 2013

26. Maurya SK, Tewari M, Sharma B and Shukla HS: Expression of procaspase 3 and activated caspase 3 and its relevance in hormone-responsive gallbladder carcinoma chemotherapy. Korean J Intern Med 28: 573-578, 2013.

27. Connolly PF, Jäger R and Fearnhead HO: New roles for old enzymes: killer caspases as the engine of cell behavior changes. Front Physiol 5: 149, 2014

28. Nilsson GM, Akhtar N, Kannius-Janson M and Baeckström D: Loss of E-cadherin expression is not a prerequisite for c-erbB2-induced epithelial-mesenchymal transition. Int J Oncol 45: 82-94, 2014

29. Ponce E, Louie MC and Sevigny MB: Acute and chronic cadmium exposure promotes E-cadherin degradation in MCF7 breast cancer cells. Mol Carcinog, 2014.

30. Sutoh Yoneyama M, Hatakeyama S, Habuchi T, et al: Vimentin intermediate filament and plectin provide a scaffold for invadopodia, facilitating cancer cell invasion and extravasation for metastasis. Eur J Cell Biol 93: 157-169, 2014.

31. Fu CH, Lin RJ, Yu J, et al: A novel oncogenic role of inositol phosphatase SHIP2 in ER-negative breast cancer stem cells: involvement of JNK/Vimentin activation. Stem Cells 32, 2048-2060, 2014. 